BOSQUE 25(3): 33-43, 2004

\title{
Eficiencia del uso del agua en plantaciones de Pinus radiata en Chile*
}

\author{
Water-use efficiency for Pinus radiata stands in Chile.
}

ANTON HUBER J., RAMIRO TRECAMAN V.

Instituto de Geociencias, Universidad Austral de Chile, Casilla 567,

Valdivia, Chile. E-mail: ahuber@uach.cl

\section{SUMMARY}

The water-use efficiency of Pinus radiata, D. Don stands in Chile was determined. The transpiration rate (net evapotranspiration / tree volumetric increase) of selected plantations with different management between the Seventh and Tenth regions of Chile were calculated. Transpiration rate ranged between 241 and $717 \mathrm{~m}^{3} / \mathrm{m}^{3}$. The smaller transpiration rate was registered in zones containing soils with a high water retention, heavy rainfall and little vegetation. The greatest rates of transpiration were determined in the plantations established in zones with less intense precipitation and a high potential evaporation. The transpiration rate was also high in sandy soils because here the trees are exposed to a prolonged water stress which restricts their growth. Precipitation, net precipitation, density of the stand, soil-water retention capacity, summer temperature, and the characteristics of the vegetation explain more than $83.8 \%\left(\mathrm{r}^{2}\right)$ of the variability in the transpiration rate between the studied plantations.

Key words: transpiration rate, Pinus radiata.

\section{RESUMEN}

Se calculó la eficiencia del uso de agua de plantaciones de Pinus radiata (D. Don) en Chile. Para ello se determinó y comparó la tasa de transpiración-evapotranspiración neta anual/incremento volumétrico fustal anual de algunas plantaciones coetáneas de Pinus radiata con distintos manejos entre la VII y X Regiones de Chile que poseen diferentes condiciones edafoclimáticas. La tasa de transpiración varió entre los 241 y $717 \mathrm{~m}^{3} / \mathrm{m}^{3}$. Los menores valores se registraron en las plantaciones establecidas en suelos arcillosos, con buena capacidad de retención de agua y con una pobre cubierta vegetal asociada, ubicadas en zonas donde predominan los eventos importantes de lluvia. Las mayores tasas de transpiración se observaron en las plantaciones establecidas en zonas con precipitaciones de menor intensidad y una alta evaporación potencial. En los arenales la tasa también fue alta, por las características de los suelos y porque los árboles estuvieron expuestos a un prolongado estrés hídrico estival, lo que restringió sus crecimientos. Las precipitaciones que alcanzaron el suelo, la densidad de las plantaciones, la capacidad de retención de agua útil de los suelos, la temperatura estival y las características de la vegetación acompañante explicaron en $83,8 \%\left(\mathrm{r}^{2}\right)$ la variabilidad de la tasa de transpiración entre las plantaciones.

Palabras clave: tasa de transpiración, Pinus radiata.

* Estudio contemplado en el Proyecto Fondecyt 1010713. 
BOSQUE 25(3): 33-43, 2004

Eficiencia del uso de agua en plantaciones de Pinus radiata en Chile

\section{INTRODUCCION}

La eficiencia del uso del agua es el rendimiento que tiene un ecosistema vegetal por unidad de agua evapotranspirada. En estudios forestales el rendimiento se expresa como la cantidad de carbono fijado o el incremento del volumen maderable o el incremento del volumen fustal de un bosque (1). Publicaciones recientes valoran el hecho de que la eficiencia del uso del agua se exprese como su valor recíproco, llamado tasa de transpiración. Ella indica la cantidad de agua evapotranspirada $\left(\mathrm{m}^{3}\right)$ para producir una unidad de volumen $\left(\mathrm{m}^{3}\right)$ de materia seca o maderable o fustal (2-4). De esta manera, la tasa de transpiración de una plantación forestal indica la cantidad de agua que fue extraída del suelo (evapotranspiración neta) (4) para producir $1 \mathrm{~m}^{3}$ de incremento fustal.

La evapotranspiración neta está regulada por las características del suelo, de la cubierta vegetal y de las condiciones climáticas. Entre las climáticas sobresalen la radiación solar, las precipitaciones, la temperatura y la humedad del aire (5-7), mientras que en las edáficas destacan, entre otras, la capacidad de retención de agua útil del suelo y la velocidad de infiltración (8). Estas dos últimas características determinan la cantidad y el tiempo que el agua del suelo está disponible para la vegetación (9). La evapotranspiración neta también está influida por la extensión y eficiencia del sistema radicular de la cubierta vegetal, la superficie foliar, la disposición y estructura de las hojas y el comportamiento de las estomas $(6,7)$.

El incremento volumétrico fustal de una plantación forestal está regulado por las condiciones del sitio, características genéticas de los árboles, del bosque y de la vegetación asociada (9-12).

El raleo de una plantación forestal afecta su desarrollo porque modifica las condiciones micrometeorológicas (13-15), aumenta la cantidad de agua que llega al suelo $(16,17)$ e incrementa la evaporación $(13,14)$. Consecuencia de esto último, la evaporación adquiere una mayor participación en la evapotranspiración neta, lo que disminuye la eficiencia de uso del agua $(4,18)$.

En general, el raleo aumenta la tasa de transpiración, porque reduce el incremento volumétrico del rodal, mientras que la evapotranspiración neta no lo hace con la misma magnitud (13).
En Chile hay más de 1.600.000 hectáreas forestadas con Pinus radiata (D. Don), distribuidas entre los 32 y $41^{\circ}$ latitud sur (19). Esta zona posee condiciones edáficas y climáticas muy diferentes que inciden en el desarrollo de las plantaciones.

Es importante conocer la relación que existe entre el consumo de agua de las plantaciones y el correspondiente incremento volumétrico y establecer la influencia que tienen las características edafoclimáticas y el manejo silvícola sobre estos resultados.

El objetivo del presente estudio es determinar y comparar la tasa de transpiración de algunas plantaciones coetáneas de Pinus radiata ubicadas entre la VII y X Regiones de Chile. Para este propósito se establecerá y analizará la relación que existe entre la cantidad de agua evapotranspirada y el incremento volumétrico fustal de cada una de ellas. Además, se determinará el efecto que tienen algunas de las características de sitio y la densidad de las plantaciones en estos resultados.

\section{MATERIAL Y METODOS}

Para establecer la tasa de transpiración de las plantaciones de Pinus radiata se determinó la cantidad de agua involucrada en la evapotranspiración neta y el incremento volumétrico fustal de algunas plantaciones entre 11 y 15 años con distinto manejo silvícola (cuadro 1). Las plantaciones se ubican en los alrededores de las ciudades de Talca, Yumbel, Collipulli y Valdivia (figura 1).

\section{CARACTERISTICAS EDAFOCLIMATICAS} DE LAS ZONAS ESTUDIADAS

PALHUEN. Las plantaciones están situadas en el fundo Palhuén ( $35^{\circ} 06^{\prime} \mathrm{S}, 72^{\circ} 00^{\prime} \mathrm{W}$ ) de propiedad de la empresa Forestal Celco S. A., distante a $55 \mathrm{~km}$ al noroeste de la ciudad de Talca (figura 1). El lugar corresponde a la zona de transición entre el secano costero y el secano interior de la provincia de Talca, VII Región. El clima local corresponde al templado cálido con lluvias invernales y estación seca prolongada (7 a 8 meses) (Csb1) (20). La precipitación media anual es de aproximadamente $1.000 \mathrm{~mm}$ (18), concentrándose el 75\% de las lluvias entre mayo y agosto (21). La temperatura media anual es de $14,7^{\circ} \mathrm{C}$, siendo enero e 

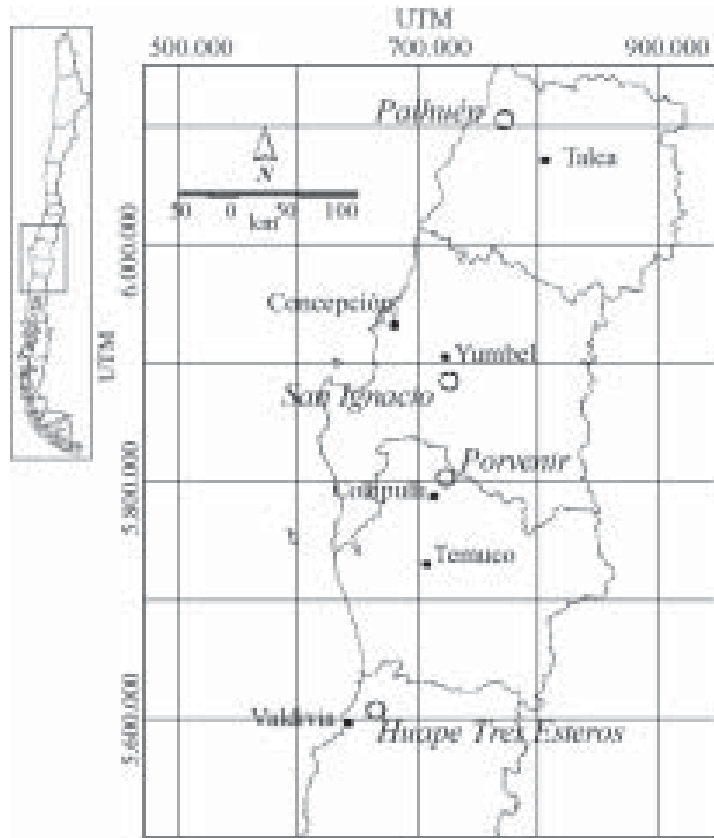

Figura 1. Zona de estudio y ubicación de las plantaciones.

The study area and location of the research sites.

mes con la temperatura media más alta $\left(18^{\circ} \mathrm{C}\right)$ y julio con la más fría $\left(10^{\circ} \mathrm{C}\right)$ (figura 2$)$.

Los suelos de la zona corresponden a la serie Constitución (22). Son suelos moderadamente profundos, formados a partir de rocas metamórficas del tipo metaesquisto o rocas sedimentarias de composición mixta (Alfisoles). Poseen textura franca arcillosa en superficie a arcillosa muy densa en profundidad, destacando la presencia de una fase moderadamente gravosa que afecta la cantidad de agua útil. La cantidad promedio de agua aprovechable de los primeros $3 \mathrm{~m}$ del perfil del suelo es de aproximadamente $16 \%$ volumen.

SAN IGNACIO. Las plantaciones están ubicadas a 13 $\mathrm{km}$ al sur de la ciudad de Yumbel, en el fundo San Ignacio (37 $14^{\prime}$ S, $72^{\circ} 19^{\prime}$ W) de la empresa Forestal Mininco S.A., VIII Región (figura 1). El clima de la zona corresponde a templado cálido con estación seca y lluviosa semejantes (Cfb) (4 a 5 meses secos) (20). La precipitación media anual de la zona es de $1.100 \mathrm{~mm}$ que tiene una distribución estacional mediterránea típica. La temperatura media anual es de $14,3^{\circ} \mathrm{C}$, enero es el mes más cálido con $19^{\circ} \mathrm{C}$ y julio el más frío con $7^{\circ} \mathrm{C}$ (23) (figura 2).

Los suelos de la zona corresponden a la serie Arenales (24) del grupo homónimo (Entisol). Se presentan en forma de llanos aluviales de baja pendiente (2\%). Su material de origen está constituido por arenas andesíticas y basálticas modernas. Son suelos moderadamente profundos, físicamente ricos en arenas (superior al 93\%) y muy pobres en arcilla. Esta característica determina altas tasas de infiltración, baja capacidad de campo y una limitada cantidad de agua aprovechable que es de aproximadamente $5 \%$ volumen en los 3 primeros metros de profundidad. Además, presentan discontinuidades litológicas, que generan perfiles muy estratificados que influyen en el movimiento interno del agua, pero por sobre todo en el desarrollo radicular. Respecto a sus características químicas, poseen un bajo contenido de nitrógeno y de materia orgánica. Además, tienen una reducida productividad natural, debido a sus características texturales y de infiltración (24).

PORVENIR. Las plantaciones están ubicadas en el fundo Porvenir $\left(37^{\circ} 55^{\prime} \mathrm{S}, 72^{\circ} 25^{\prime} \mathrm{W}\right)$ de la empresa Forestal Mininco S.A., IX Región, ubicado a $11 \mathrm{~km}$ al nordeste de la ciudad de Collipulli (figura 1). El clima de la zona corresponde a templado cálido con estación seca y lluviosa semejantes (Cfb) (20). La precipitación media anual es de $1.200 \mathrm{~mm}$ (23) con una distribución estacional bien marcada (figura 2 ). La temperatura media anual es de $13,1^{\circ} \mathrm{C}$, la media mensual máxima se registra en enero con $18^{\circ} \mathrm{C}$ y la mínima en junio con $7^{\circ} \mathrm{C}(23)$.

Los suelos de la zona corresponden a la serie Collipulli (24), que pertenecen al grupo de los suelos rojo arcillosos (Alfisoles). Son suelos desarrollados a partir de material piroclástico antiguo que descansa sobre conglomerados volcánicos muy meteorizados y que no guardan relación genética con el perfil. Se manifiestan en forma de lomajes suaves a quebrados. Su textura es franco arcillosa a arcillosa y la estructura es granular a subpoliédrica. De color pardo rojizo a rojizo, son suelos moderadamente profundos a profundos, muy plásticos, poseen una gran cantidad de agua aprovechable, la que aumenta en profundidad. La cantidad de agua aprovechable en los primeros 3 metros es de aproximadamente 28\%/volumen (24). 
BOSQUE 25(3): 33-43, 2004

Eficiencia del uso de agua en plantaciones de Pinus radiata en Chile

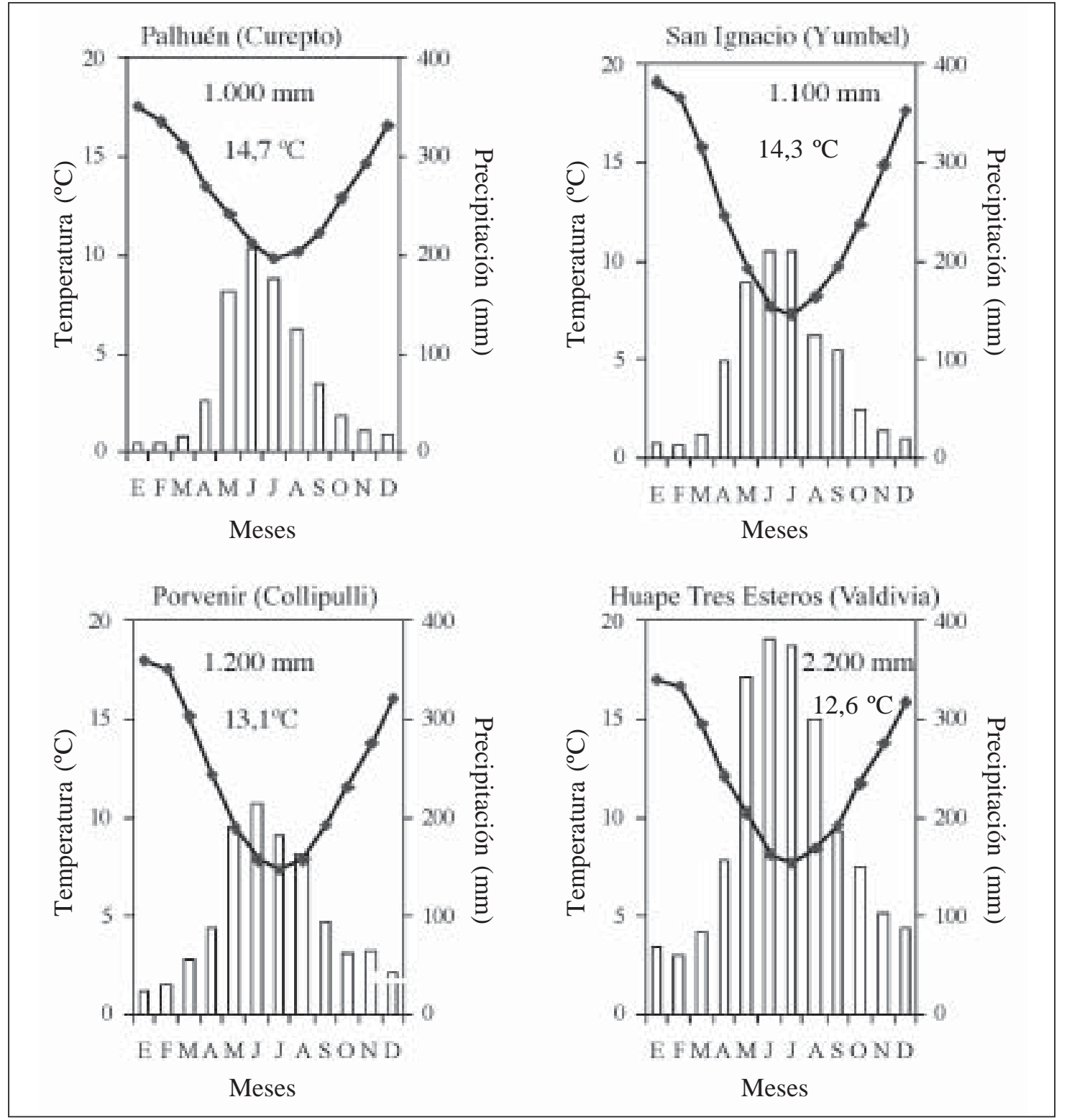

Figura 2. Climograma de las localidades consideradas en el estudio.

Climatic characteristics of the sample areas.

(Fuente: 23, 26)

El drenaje interno se presenta lento, con rangos que van desde muy lentos a moderados (25).

HUAPE TRES ESTEROS. La plantación está ubicada en el fundo Huape Tres Esteros (3948' S, 73 $14^{\prime}$ W) de la Universidad Austral de Chile, X Región (figura 1). La zona posee un clima templado lluvioso con influencia mediterránea (Cfsb) (20). La temperatura media anual es de $12,6^{\circ} \mathrm{C}$. Enero es el mes más cálido con $17^{\circ} \mathrm{C}$ y julio el más frío con $7^{\circ} \mathrm{C}$ (figura 2 ). La precipitación media anual de la zona es de $2.200 \mathrm{~mm}$ y presenta una fuerte oscilación estacional (julio 400, febrero $60 \mathrm{~mm}$ ) (26).

El suelo corresponde a la serie Correltué (22), del grupo de los suelos trumaos, el cual se ha desarrollado a partir de cenizas volcánicas modernas depositadas sobre el complejo metamórfi- 
co de la Cordillera de la Costa (Ultisoles). Son suelos profundos de color pardo oscuro, presentan una textura moderadamente fina hasta los 60 $\mathrm{cm}$ y muy fina en profundidades mayores. Son suelos blandos, friables, ligeramente plásticos y adhesivos en profundidad, la cantidad de agua aprovechable del suelo de la plantación es de un $20 \% /$ volumen promedio en los primeros $3 \mathrm{~m}$ del perfil.

Para determinar la evapotranspiración de cada plantación se delimitó en ella una parcela de 100x100 m, donde se instalaron los diferentes equipos e instrumentos. La evapotranspiración neta $($ Evtrn = agua extraída sólo del suelo $)$ y la total (Evtrt $=$ evapotranspiración neta más pérdidas de agua por intercepción) se calculó con la metodología del balance hídrico, basada en la ecuación de continuidad de Feller (27):

$$
\begin{aligned}
& \text { Evtrn }=\mathrm{Pp}-(\mathrm{Ic}+\Delta \mathrm{W}+\mathrm{A}+\mathrm{Per}) \\
& \text { Evtrt }=\operatorname{Pp}-(\Delta \mathrm{W}+\mathrm{A}+\mathrm{Per})
\end{aligned}
$$

La precipitación incidente $(\mathrm{Pp})$ fue registrada con dos pluviógrafos ubicados a campo abierto, en terrenos aledaños a cada una de las plantaciones.

La cantidad de agua retenida por la biomasa aérea del bosque (pérdidas de agua por intercepción del dosel) fue calculada con la siguiente ecuación [3]:

$$
\mathrm{Ic}=\mathrm{Pp}-(\mathrm{Pd}+\mathrm{Pf})
$$

donde:

Ic : pérdidas de agua por intercepción (mm)

$\mathrm{Pp}$ : precipitación incidente $(\mathrm{mm})$

Pd : precipitación directa $(\mathrm{mm})$

Pf : escurrimiento fustal (mm)
Las precipitaciones que atravesaron el dosel de las plantaciones para alcanzar el suelo (precipitación directa) fueron medidas con una canaleta metálica en forma de $\mathrm{V}$, de $15 \mathrm{~cm}$ de ancho y 25 $\mathrm{m}$ de largo, ubicada a $30 \mathrm{~cm}$ sobre el suelo. La lluvia que utilizó el tronco de los árboles como senda de fluido para llegar al suelo (escurrimiento fustal) fue recolectada con collarines de goma sellados alrededor del tronco de 15 árboles por parcela. El agua recogida en cada caso fue conducida a un estanque provisto con un sistema de registro automático.

La variación temporal del contenido de agua del suelo $(\Delta \mathrm{W})$ se determinó hasta los $3 \mathrm{~m}$ de profundidad con una sonda de neutrones (Troxler 4300). Se acordó esta profundidad porque el desarrollo radicular de las distintas plantaciones no sobrepasó los 2,3 m (18, 28-31).

Las mediciones fueron realizadas mensualmente en 15 tubos enterrados en el suelo de cada plantación. Los tubos estaban ubicados sobre y entre las hileras de plantación para reducir al máximo los errores asociados a la desigual distribución del agua en el suelo de los bosques (18). La calibración de este dispersor de neutrones se hizo en terreno de acuerdo a lo propuesto por Brechtel (32). La escorrentía superficial (E) fue despreciada porque todas las plantaciones están ubicadas en terrenos con una pendiente inferior al $2 \%$.

La percolación (Per) fue considerada equivalente a la cantidad de agua que atravesó el suelo a una profundidad preestablecida de $3 \mathrm{~m}$. Esta agua fue recogida a dicha profundidad mediante 4 bandejas de $30 \times 30 \mathrm{~cm}$ por parcela. Cada bandeja estuvo conectada a un recipiente enterrado al cual drenaba el agua recolectada. Mensualmente, y con ayuda de una bomba portátil, se extrajo el agua y se determinó su volumen.

Para determinar los incrementos volumétricos de las plantaciones, mensualmente se midió el DAP y la altura de 25 árboles de cada parcela. Con esta información se determinó mensualmente el volumen de cada árbol, utilizando para ello las funciones locales de volumen de las empresas propietarias de las plantaciones. Además, en cada parcela se instaló un termógrafo electrónico $\left(\mathrm{Hobo}^{\circledR}\right)$ para registrar la temperatura del aire por ser un parámetro meteorológico importante en la regulación de la evapotranspiración. 
BOSQUE 25(3): 33-43, 2004

Eficiencia del uso de agua en plantaciones de Pinus radiata en Chile

\section{RESULTADOS Y DISCUSION}

En el cuadro 1 se presentan las características de las distintas plantaciones y las tasas de transpiración anual calculadas con la evapotranspiración neta (Evtrn) y total (Evtrt).

Los mayores consumos de agua por evapotranspiración neta se registraron en las zonas más lluviosas, establecidas en suelos con alta capacidad de retención de agua útil y donde la sobrevivencia de la cubierta herbácea acompañante fue más prolongada (cuadro 1, figura 2). En consecuencia, los mayores valores se registraron en las plantaciones ubicadas en las zonas de Valdivia y Collipulli.

Los valores máximos de evapotranspiración neta en estas localidades se lograron en los años que tuvieron una primavera-verano más húmeda. Estas condiciones pluviométricas generaron una mayor disponibilidad temporal de agua para el consumo de los árboles y permitieron una sobrevivencia más prolongada de la cubierta herbácea. Esta situación, extendió la participación de la cubierta herbácea en la evapotranspiración neta de la plantación.

La menor evapotranspiración neta se registró en los Arenales, debido a las reducidas precipitaciones estivales y a la baja capacidad de retención de agua útil de los suelos (24). Estas condiciones disminuyeron la disponibilidad de agua para las plantaciones y la cubierta herbácea. En consecuencia, la vegetación acompañante de las plantaciones redujo su período de sobrevivencia y con ello el tiempo de su participación en la evapotranspiración neta (12).

De acuerdo a estos resultados, la distinta evapotranspiración neta anual entre las plantaciones de las diferentes zonas se explica principalmente por la desigual cantidad y distribución temporal de las precipitaciones, la disímil capacidad de retención de agua útil de los suelos y no tanto por las condiciones meteorológicas que afectan la evapotranspiración. La importancia de la capacidad de retención de agua de los suelos en la cantidad anual de agua evapotranspirada por plantaciones forestales, ya había sido observada por Abrahamson et al. (8) y por Huber y Trecaman (12). En suelos con estas características, estos autores identificaron un período de baja evapotranspiración asociada a la reducida cantidad de agua en el suelo, el que coincidía con los meses de mayor demanda de agua por evaporación potencial.

Sorprende que la densidad de las plantaciones no tuviera mayor incidencia en la evapotranspiración neta en Palhuén, San Ignacio y Porvenir (cuadro 1). Ello se debió, principalmente, a que al final del período deficitario en precipitaciones la mayor parte de la reserva de agua de los suelos había sido consumida. En consecuencia, sólo varió la fecha en que se llegó a esta situación. Estos resultados concuerdan con diversos estudios que señalan que el mayor impacto hidrológico de la reducción de densidad de una plantación forestal son las menores pérdidas de agua por intercepción y no los cambios en la cantidad de agua involucrada en la evapotranspiración neta para estas condiciones climáticas (12-16).

Se registraron diferencias importantes en el incremento volumétrico de las plantaciones entre años y entre las localidades (cuadro 1). Los mayores incrementos se registraron en años que tuvieron una primavera-verano más húmeda, en plantaciones establecidas en suelos con una superior capacidad de retención de agua útil y ubicadas en zonas con condiciones climáticas más favorables para el crecimiento.

En los Arenales, debido al persistente estrés hídrico al que estuvieron expuestas las plantaciones durante los meses en que la temperatura era la más favorable para el crecimiento y a la baja fertilidad de estos suelos, los árboles registraron un bajo incremento volumétrico (cuadro 1).

Cuando se ralearon las plantaciones, los árboles restantes tuvieron una mayor cantidad de agua disponible, lo que incidió positivamente en su crecimiento (cuadro 1). El mayor desarrollo individual de los árboles de las plantaciones más ralas en algunos casos fue suficiente para igualar el incremento volumétrico de aquellas que mantuvieron su densidad inicial (cuadro 1). Robinson et al. (3) y Woodruff et al. (15) obtuvieron resultados similares en plantaciones de Pinus baksiana y Pseudotsuga menziesii, respectivamente; pero recalcaron la importancia que tiene la densidad final en estos incrementos volumétricos.

Para generar un incremento fustal de un $\mathrm{m}^{3}$ en la plantación de Pinus radiata, ubicada cerca de Valdivia, durante dos años consecutivos, se utilizaron 409 y $496 \mathrm{~m}^{3} / \mathrm{año}$ de agua, respectivamente. La distinta evapotranspiración anual se debió a la diferente distribución temporal de las precipita- 
BOSQUE 25(3): 33-43, 2004

Eficiencia del uso de agua en plantaciones de Pinus radiata en Chile

\section{CUADRO 1}

Características de sitio y de las plantaciones de Pinus radiata estudiadas. Precipitación, evapotranspiración neta y total, incremento volumétrico y tasa de transpiración calculada con la evapotranspiración neta (Tasa $\left.{ }^{1}\right)$ y con la evapotranspiración total $\left(\right.$ Tasa $\left.^{2}\right)$.

Sampling areas and stand characteristics. Precipitation, net and total evapotranspiration, volume increase and transpiration rate calculated with net evapotranspiration $\left(\mathrm{Tasa}^{1}\right)$ and total evapotranspiration $\left(\mathrm{Tasa}^{2}\right)$

\begin{tabular}{|c|c|c|c|c|c|c|c|c|c|c|c|c|c|c|}
\hline \multicolumn{6}{|c|}{ Características de las plantaciones } & \multicolumn{9}{|c|}{ Resultados } \\
\hline \multirow[b]{2}{*}{$\mathrm{L}$} & \multirow[b]{2}{*}{$\mathrm{E}$} & \multirow[b]{2}{*}{ Den } & \multirow[b]{2}{*}{ Ts } & \multicolumn{2}{|c|}{ Vegetación } & \multirow[b]{2}{*}{$\mathrm{Pp}$} & \multirow[b]{2}{*}{ Pn } & \multirow[b]{2}{*}{$\mathrm{Pv}$} & \multirow[b]{2}{*}{$\mathrm{Tv}$} & \multirow[b]{2}{*}{ Evtrn } & \multirow[b]{2}{*}{ Evtrt } & \multirow[b]{2}{*}{ Vol } & \multirow[b]{2}{*}{ Tasa $^{1}$} & \multirow[b]{2}{*}{ Tasa $^{2}$} \\
\hline & & & & tipo & Per & & & & & & & & & \\
\hline 1 & 12 & 700 & M & h1 & 2 & 1.271 & 838 & 171 & 15,4 & 545 & 978 & 8 & 717 & 1.287 \\
\hline 1 & 12 & 1.250 & M & h1 & 2 & 1.271 & 786 & 171 & 15,4 & 578 & 1.063 & 10 & 556 & 1.022 \\
\hline 2 & 14 & 796 & A & h1 & 6 & 1.133 & 742 & 141 & 16,8 & 422 & 813 & 10 & 435 & 838 \\
\hline 2 & 15 & 796 & A & h1 & 6 & 1.110 & 687 & 117 & 15,5 & 412 & 834 & 9 & 448 & 907 \\
\hline 2 & 12 & 1.206 & A & h1 & 9 & 1.238 & 866 & 322 & 17,1 & 466 & 838 & 12 & 376 & 676 \\
\hline 2 & 12 & 549 & A & h1 & 9 & 1.238 & 1.015 & 322 & 17,1 & 449 & 672 & 13 & 345 & 517 \\
\hline 2 & 13 & 1.206 & A & h1 & 6 & 1.199 & 948 & 89 & 14,4 & 277 & 528 & 9 & 315 & 600 \\
\hline 2 & 13 & 549 & A & h1 & 6 & 1.199 & 1.008 & 89 & 14,4 & 293 & 484 & 9 & 329 & 544 \\
\hline 2 & 14 & 1.206 & A & h1 & 9 & 1.276 & 979 & 268 & 16,9 & 432 & 729 & 11 & 389 & 657 \\
\hline 2 & 14 & 549 & A & h1 & 9 & 1.276 & 1.030 & 208 & 16,9 & 412 & 658 & 11 & 381 & 609 \\
\hline 3 & 12 & 833 & RA & h2 & 9 & 1.090 & 714 & 153 & 17,3 & 544 & 920 & 16 & 338 & 571 \\
\hline 3 & 13 & 833 & RA & h2 & 9 & 1.288 & 813 & 134 & 17,3 & 581 & 1.056 & 21 & 281 & 510 \\
\hline 3 & 11 & 833 & RA & h2 & 12 & 1.256 & 938 & 236 & 14,3 & 584 & 902 & 24 & 241 & 373 \\
\hline 3 & 11 & 395 & RA & h2 & 12 & 1.256 & 1.023 & 236 & 14,3 & 613 & 846 & 19 & 319 & 441 \\
\hline 3 & 12 & 833 & RA & h2 & 12 & 1.219 & 877 & 344 & 16,9 & 612 & 954 & 22 & 282 & 440 \\
\hline 3 & 12 & 395 & RA & $\mathrm{h} 2$ & 12 & 1.219 & 961 & 344 & 16,9 & 659 & 917 & 17 & 379 & 527 \\
\hline 3 & 13 & 833 & RA & h2 & 10 & 1.321 & 959 & 239 & 15,8 & 630 & 992 & 19 & 326 & 514 \\
\hline 3 & 13 & 395 & RA & h2 & 10 & 1.321 & 1.022 & 239 & 15,8 & 601 & 900 & 16 & 388 & 581 \\
\hline 4 & 13 & 830 & $\mathrm{~T}$ & a3 & 12 & 1.943 & 1.628 & 404 & 12,4 & 618 & 933 & 15 & 409 & 618 \\
\hline 4 & 14 & 830 & $\mathrm{~T}$ & $\mathrm{a} 3$ & 12 & 2.219 & 1.903 & 443 & 14,8 & 694 & 1.010 & 14 & 496 & 721 \\
\hline
\end{tabular}

Lugar de establecimiento de las plantaciones (L), edad (E: años), densidad (Den: árboles/ha), tipo de suelo (Ts) tipo y persistencia de la cubierta vegetal asociada (Per: meses), monto de las precipitaciones (Pp: mm/año), precipitación neta (Pn: mm/año), precipitación total septiembre-marzo (Pv: mm), temperatura media septiembremarzo (Tv: $\left.{ }^{\circ} \mathrm{C}\right)$, evapotranspiración neta (Evtrn: mm/año) y evapotranspiración total (Evtrt: mm/año); incremento volumétrico fustal (Vol: $\left.\mathrm{m}^{3} / \mathrm{a}\right)$ y tasa de transpiración calculada con la evapotranspiración neta $\left(\operatorname{Tasa}^{1}: \mathrm{m}^{3} / \mathrm{m}^{3}\right)$ y con la evapotranspiración total $\left(\mathrm{Tasa}^{2}: \mathrm{m}^{3} / \mathrm{m}^{3}\right)$.

Sampling areas (L), age (E: years), density (Den: trees/ha), soil type (Ts), type and permanence of grass and associated shrubs (Per: months), precipitation (Pp: mm/year), net precipitation (Pn: mm/year), September to March precipitation (Pv: $\mathrm{mm}$ ), September to March average temperature $\left(\mathrm{Tv}:{ }^{\circ} \mathrm{C}\right)$, net evapotranspiration (Evtrn: mm/year) and total evapotranspiration (Evtrt: $\mathrm{mm} / \mathrm{year}$ ); volume increase (Vol: $\mathrm{m}^{3} / \mathrm{a}$ ) and transpiration rate calculated with net evapotranspiration (Tasa ${ }^{1}$ ) and total evapotranspiration $\left(\mathrm{Tasa}^{2}\right)$.

Referencia
Localidad
Suelo (serie - grupo - capacidad de agua útil)
1: Palhuén
M : Constitución, metamórfico, $16 \%$ vol
2: San Ignacio
3: Porvenir
A : Arenales, arenosos, $8 \%$ vol
RA : Collipulli, rojo arcillosos, $25 \%$ vol
4: Huape Tres Esteros
T : Correltué, trumao, $20 \%$ vol 
BOSQUE 25(3): 33-43, 2004

Eficiencia del uso de agua en plantaciones de Pinus radiata en Chile

Características de la vegetación asociada al bosque

Tipo vegetación asociada Densidad cubierta vegetal (\% cobertura)

h: herbácea $\quad 0$ muy rala $(<25 \%)$

a: arbustiva 1 rala $(26-50 \%)$

2 densa $(51-70 \%)$

3 muy densa $(>71 \%)$

ciones durante este período (cuadro 1) (33). Por consiguiente, el suelo tuvo una distinta disponibilidad de agua que pudieron aprovechar los árboles durante los dos años. Además, esta distribución temporal repercutió sobre la extensión del período de sobrevivencia de la cubierta herbácea acompañante (cuadro 1). Consecuencia de ello, el suelo y la vegetación herbácea tuvieron una distinta participación en la evapotranspiración neta durante estos dos años, lo que modificó la tasa de transpiración (cuadro 1). El efecto que tuvo la cubierta vegetal acompañante en la eficiencia de uso del agua es coincidente con los resultados mencionados por Longpré et al. (34) y Mohammed et al. (35).

Cuando la tasa de transpiración para la zona de Valdivia se calculó con la evapotranspiración total, la cantidad de agua requerida para generar 1 $\mathrm{m}^{3}$ de biomasa fustal durante los dos años ascendió a 618 y $721 \mathrm{~m}^{3}$, respectivamente.

En la zona de los Arenales la tasa de transpiración calculada con la evapotranspiración neta osciló entre los 314 y 455. Estos valores fueron superiores a los calculados para las plantaciones ubicadas en suelos rojo arcillosos (cuadro 1). Ello se explica parcialmente por el menor desarrollo que tuvieron los árboles debido a la baja fertilidad de estos suelos (24). Otro factor a considerar es el estrés hídrico casi permanente al que están expuestas estas plantaciones durante el estío, lo que exige un gasto energético adicional a los árboles para acceder a la escasa cantidad de agua del suelo. Esta aseveración coincide con los resultados de Dixon (11) y Huber y Trecaman (12). A pesar de que la evapotranspiración neta fue inferior que en las otras localidades, la tasa de transpiración se mantuvo alta debido principalmente al muy bajo incremento volumétrico fustal (cuadro 1). Al calcular la tasa de transpiración en los Arenales con la evapotranspiración total, las cantidades de agua para producir un $\mathrm{m}^{3}$ de incremento fustal aumentaron, oscilando entre los 544 y $907 \mathrm{~m}^{3}$.
La mejor eficiencia del uso de agua se registró en las plantaciones establecidas en los suelos rojo arcillosos. Estos suelos, con una buena capacidad de retención de agua útil, más las favorables condiciones climatológicas de la zona (figura 2), permitieron que los árboles tuvieran un mayor crecimiento (cuadro 1). A su vez, en años con escasas precipitaciones estivales, la cubierta herbácea no sobrevivió durante el verano. Bajo esta circunstancia, la transpiración de las herbáceas durante el estío fue mínima y la competencia por agua entre los árboles y la vegetación asociada dejó de existir. Esta situación mejoró la eficiencia del uso de agua de las plantaciones. La tasa de transpiración aumentó en los años que tuvieron un período estival más lluvioso. Ello se explica porque las precipitaciones durante el verano, al ser generalmente menos intensas, humedecen sólo la superficie y son rápidamente evaporadas. Esta situación permitió que la cubierta herbácea sobreviviera por más tiempo (12). Bajo estas circunstancias, la tasa de transpiración de las plantaciones volvió a aumentar, porque una cantidad indeterminada de agua se reintegró a la atmósfera sin que pudiera ser aprovechada por los árboles $(8,12)$.

Las mayores tasas de transpiración se registraron en las plantaciones ubicadas cerca de Curepto (cuadro 1). Ello se explica por las características de las precipitaciones y las condiciones meteorológicas que favorecen la evaporación (figura 2). En esta zona predominan, durante el estío, las precipitaciones de poca intensidad (21), que rápidamente se reintegran a la atmósfera por evaporación. Además, el humedecimiento superficial del suelo permitió que la cubierta herbácea participara por un tiempo más prolongado en la evapotranspiración neta, aumentando la tasa de transpiración.

Durante el año de mediciones las precipitaciones de Palhuén sobrepasaron en más de un $27 \%$ al promedio, lo que incrementó la evaporación del suelo y la transpiración de la cubierta herbácea, aumentando con ello la tasa de transpiración. 
En años con una distribución normal de las precipitaciones, el horizonte superficial debería estar más seco y la cubierta herbácea tener una menor permanencia, lo que en conjunto debería disminuir la tasa de transpiración.

Cuando la tasa de transpiración en la zona de Curepto se calculó con los valores de la evapotranspiración total, la cantidad de agua necesaria para producir un $\mathrm{m}^{3}$ de incremento fustal sobrepasó los $1.000 \mathrm{~m}^{3}$ (cuadro 1). Estos fueron los valores más altos de todas las zonas estudiadas, los que indican la peor eficiencia del uso del agua. Estos resultados se debieron principalmente a las características de las precipitaciones de este año en particular y a las altas pérdidas de agua por intercepción, las que fueron equivalentes aproximadamente al $40 \%$ de la precipitación total (cuadro 1).

El análisis de regresión múltiple paso a paso (Stepwise Durbin Watson) entre la cantidad anual de agua involucrada en la evapotranspiración neta de las plantaciones y las variables: densidad de las plantaciones, capacidad de retención de agua útil de los suelos, características de la vegetación acompañante, precipitación neta anual, precipitación anual y temperatura promedia máxima de los períodos septiembre-marzo explicaron en un $83,8 \%\left(\mathrm{r}^{2}\right)$ la variabilidad de la tasa de transpiración entre las zonas estudiadas (cuadro 2, 3 y figura 3 ).

\section{CUADRO 2}

Regresión múltiple paso a paso (Stepwise Durbin Watson) para identificar los factores que explican la variabilidad de la tasa de transpiración de Pinus radiata en Chile.

Multiple regression step by step (Stepwise Durbin Watson) to identify the factors that explain the variability in the transpiration rate of Pinus radiata stands in Chile.

\begin{tabular}{|l|c|c|c|c|}
\hline \multicolumn{5}{|c|}{ Variable dependiente: tasa de transpiración calculada con evapotranspiración neta $\left(\mathrm{m}^{3} / \mathrm{m}^{3}\right)$} \\
\hline Parámetro & Estimador & Error estándar & $\mathrm{t}$ & Valor p \\
\hline Constante & $-185,604$ & 228,647 & $-0,811751$ & 0,4275 \\
Densidad plantación (árboles/ha) & $-0,160142$ & 0,0500409 & $-3,20022$ & 0,0050 \\
Agua útil (\% vol) & $-19,7091$ & 8,22089 & $-2,39744$ & 0,0276 \\
Densidad cubierta herbácea (\%) & 4,54526 & 3,62439 & 1,25408 & 0,2259 \\
Precipitación neta (mm/año) & $-0,630788$ & 0,162018 & $-3,89333$ & 0,0011 \\
Precipitación anual (mm/año) & 0,722275 & 0,263881 & 2,73712 & 0,0135 \\
$\mathrm{~T}^{\mathbf{o}}$ media de verano ( $\left.{ }^{\circ} \mathrm{C}\right)$ & 30,492 & 8,76072 & 3,48053 & 0,0027 \\
\hline
\end{tabular}

CUADRO 3

ANDEVA de la regresión múltiple utilizada para explicar la variabilidad de la tasa de transpiración de Pinus radiata en Chile.

ANOVA of multiple regression used to explain the variability of the transpiration rate of Pinus radiata stands in Chile.

\begin{tabular}{|l|c|c|c|l|l|}
\hline $\begin{array}{l}\text { Fuente } \\
\text { de variación }\end{array}$ & $\begin{array}{c}\text { Suma } \\
\text { de cuadrados }\end{array}$ & $\begin{array}{c}\text { Grados } \\
\text { de libertad }\end{array}$ & $\begin{array}{c}\text { Cuadrado de } \\
\text { la media }\end{array}$ & Test F & Valor p \\
\hline Modelo & $263.674,0$ & 4 & $43.945,60$ & 15,61 & 0,0000 \\
Residual & $50.673,3$ & 16 & $2.815,18$ & & \\
Total & $314.347,0$ & 24 & & \\
\hline
\end{tabular}

$r^{2}=83,8798 \%$

Error estándar de estimación $=53,0583$

Error medio absoluto $=37,0123$

Test de Durbin-Watson $=1,82466$ 


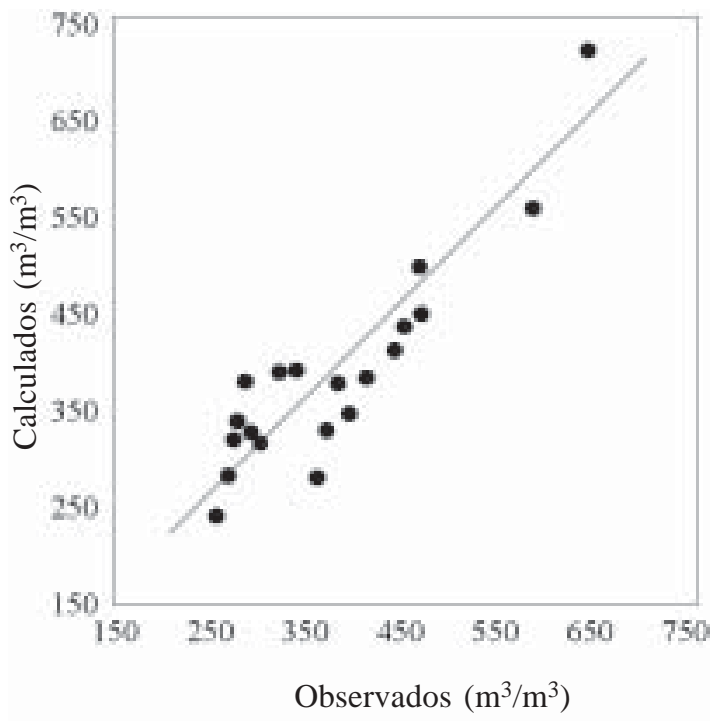

Figura 3. Relación entre la tasa de transpiración medida y calculada.

The relationship between measured or estimated transpiration rates.

La variabilidad de la tasa de transpiración para las distintas localidades calculada con la evapotranspiración neta tuvo un mayor coeficiente de determinación que la determinada con la evapotranspiración total anual $\left(\mathrm{r}^{2}=0,78\right)$, porque la primera sólo considera la cantidad de agua que efectivamente fue extraída desde el suelo (cuadro 3 y figura 3 ). La evapotranspiración total incluye el agua interceptada por el dosel, la que no es aprovechada por los árboles y, por lo tanto, no participa en su desarrollo $(5,8)$

\section{CONCLUSIONES}

La cantidad de agua necesaria para producir un $\mathrm{m}^{3}$ de volumen fustal en las plantaciones de Pinus radiata en la zona centro sur de Chile osciló entre los 241 y $717 \mathrm{~m}^{3}$. Estos valores estuvieron influidos por la densidad de las plantaciones, temperatura estival y por las características del suelo y vegetación acompañante, que explicaron en más de un $83 \%$ la variabilidad de la tasa de transpiración entre las plantaciones. Las menores tasas de transpiración estuvieron asociadas a suelos con buena capacidad de retención de agua, eventos importantes de lluvia y vegetación acompañante de menor permanencia durante el año
Las mayores tasas de transpiración se registraron en zonas con suelos de baja capacidad de almacenamiento de agua, condiciones meteorológicas favorables para la evaporación y un régimen de precipitaciones que se caracterizó por eventos de lluvia de poco aporte de agua y baja intensidad.

Las plantaciones forestales requieren una gran cantidad de agua para su desarrollo, por consiguiente, su impacto sobre la disponibilidad del recurso agua es especialmente importante en las zonas menos lluviosas de Chile.

\section{AGRADECIMIENTOS}

Los autores agradecen a las empresas Forestal CELCO S.A., Forestal MININCO S.A. y CEFOR de la Facultad de Ciencias Forestales de la Universidad Austral de Chile por su importante colaboración en el desarrollo de esta investigación, y al Prof. Víctor Figueroa del Instituto de Estadística de la Universidad Austral de Chile, por la revisión crítica de los análisis estadísticos realizados en este trabajo.

\section{BIBLIOGRAFIA}

(1) SANDS, R., D. MULLIGAN. Water and nutrient dynamics and tree growth. Forest Ecology and Management, 1990, $\mathrm{N}^{\circ}$ 30, p. 91-111.

(2) BRADY, N., R. WEIL. The Nature and Properties of Soils. 20th ed, New Jersey, USA: Prentice-Hall, Inc. Simon \& Schuster. 1999. 881 p.

(3) ROBINSON, D. E., R. G. WAGNER, F. W. BELL C. J. SWANTON. Photosynthesis, nitrogen-use efficiency, and water-use efficiency of jack pine seedlings in competition with four boreal forest plant species. Canadian Journal of Forest Research, 2001, $\mathrm{N}^{\mathrm{o}} 31$, p. 2014-2025.

(4) EASTHAM, J., C. W. ROSE, D. CHARLES-EDWARS D. CAMERON, S. RANCE. Planting density effects on water use efficiency of trees and pasture in an agroforestry experiment. New Zealand Journal of Forestry Science, 1990, vol. 20, No 1 , p 39-53.

(5) CALDER, I.R. Water use by forests, limits and controls. Tree Physiology, 1998, No 18, p 625-631.

(6) HUBER, A., G. GARCIA. Importancia de los factores meteorológicos en la transpiración potencial de Pinus radiata. $\phi Y T O N, 1999, \mathrm{~N}^{\circ} 65$, p. 143-152.

(7) MEINZER, F C M. J CLEARWATER., G. GOLDSTEIN. Water transport in trees: current perspectives, new insights and some controversies. Environmental and Experimental Botany, 2001, $\mathrm{N}^{\circ} 45$, p. 239-262.

(8) ABRAHAMSON, D. A., P. M. DOUGHERTY, S. J. ZARNOCH. Hydrological components of a young loblolly pine plantation on a sandy soil with estimates $\mathrm{pf}$ water use and loss. Water Resources Research, 1998 vol. $34, \mathrm{~N}^{\circ} 12$. p. $3.503-3.513$. 
(9) GERDING, V , J E E SCHLATTER, Variables y factores del sitio de importancia para la productividad de Pinus radiata D. Don en Chile. Bosque (Chile), 1995, vol. $16, \mathrm{~N}^{\circ} 2$, p. $39-56$.

(10) TESKEY, R. O., D. W. SHERIFF. Water use by Pinus radiata trees in a plantation. Trees Physiology, 1995, $\mathrm{N}^{\circ} 16$, p. $273-279$

(11) DIXON, R. K. Physiological processes and tree growth In: DIXON, R. K, R.S. MELDAHL, G. A. RUARK W. G. WARREN. Process modeling of forest growth responses of environmental stress. Portland: Timber Press Inc., USA. 1998. p. 81-95.

(12) HUBER, A., R. TRECAMAN. Efecto de la variabilidad interanual de las precipitaciones sobre el desarrollo de las plantaciones de Pinus radiata (D. Don) en la zona de los arenales VIII Región, Chile. Bosque (Chile), 2002 vol. $23 \mathrm{~N}^{\circ} 2$, p. $43-49$

(13) BREDA N, A. GRANIER, G. AUSSENAC. Effects of thinning on soil and tree water relations, transpiration and groth in an oak forest (Quercus petraea (Matt.) Lieb.). Tree Physiology, 1995, No 15, p.295-306.

(14) TANG, Z., J. L. CHAMBERS, S. GUDDANTI, J. P. BARNETT. Thinning, fertilization, and crown position interact to control physiological responses of loblolly pine. Tree Physiology, 1999, No 19 , p. 87-94.

(15) WOODRUFF, D. R., B. J. BOND, G. A. RITCHIE, W. SCOTT. Effects of stand density on the growth of young Douglas-fir trees. Canadian Journal of Fores Research, 2002, No 32, p. 420-427.

(16) MEADOWS J. S., J. C. GOELZ. Fifth-Year Response to Thinning in a Water Oak Plantation in North Louisiana. Southern Journal of Applied Forestry, 2001, vol. $25, \mathrm{~N}^{\mathrm{o}} 1$, p. $31-39$.

(17) CROCKFORD, R. H., D. P. RICHARDSON. Partitioning of rainfall into throughfall, stemflow and interception: effect of forest type, ground cover and climate. Hydrological Processes, 2000, $\mathrm{N}^{\circ} 14$, p. 2.903-2.920.

(18) HUBER, A., R. TRECAMAN. Efecto de las características de una plantación de Pinus radiata en la distribución espacial del contenido de agua edáfica. Bosque (Chile), 2000, vol. 21, No 1, p. 37-44.

(19) INSTITUTO FORESTAL (INFOR). Estadísticas Forestales 84. Santiago, Chile. 2001. 145 p.

(20) FUENZALIDA, H. Clima: Geografía Económica de Chile. Santiago: Corporación de Fomento de la Producción. Chile. 1971, p. 99-192.

(21) FORESTAL CELCO. Base de datos meteorológicos. Publicación periódica. Constitución. 2002. 3 p.

(22) INSTITUTO DE INVESTIGACION DE RECURSOS NATURALES (IREN). Suelos: descripciones proyecto aerofotogramétrico Chile/O.E.A./B.I.D. Chile. 1964. $391 \mathrm{p}$.

(23) MINISTERIO DE OBRAS PUBLICAS (MOP). Balance Hídrico de Chile. Santiago: Dirección General de Aguas, Chile. 1987. 12 p.
(24) CARRASCO, P , J. MILLAN, L. PEÑA Suelos de la cuenca del río Biobío. Características y problemas de uso. Serie Análisis territorial. Ediciones Universidad de Concepción. 1993. 107 p.

(25) BONELLI, C., J. SCHLATTER. Caracterización de suelos rojo arcillosos de la zona Centro-Sur de Chile. Bosque (Chile), 1995, vol. 16, $\mathrm{N}^{\circ}$ 2, p. 21-37.

(26) HUBER, A. Diez años de observaciones climatológicas en la Estación Teja-Valdivia (Chile) 1960-1969. Instituto de Geología y Geografía, Universidad Austral de Chile. 1970. $61 \mathrm{p}$

(27) FELLER, M. Water balance in Eucalyptus regnans, $E$ oblicua, y Pinus radiata forest in Victoria. Australian Forestry, 1981, vol. 44, No 3, p. 153-161.

(28) MOLINA, C. Influencia de una plantación joven de Pinu radiata y Eucalyptus globulus en la redistribución de la precipitaciones y humedad del suelo. Facultad de Filosofía y Humanidades, Universidad Austral de Chile. 1997. $84 \mathrm{p}$.

(29) SANDOVAL, J. El efecto de la redistribución de las raíces de una plantación de Pinus radiata (D. Don) y una pradera natural en el balance hídrico en un suelo rojo arcilloso de la zona de Collipulli (IX región). Tesis Ing. Forestal. Facultad de Ciencias Forestales, Universidad Austral de Chile. 1998. 89 p.

(30) ULLOA, C. Influencia del manejo sobre el balance hídrico en plantaciones de 14 años de edad en Pinus radiata (D.Don) en Los Ángeles, VIII Región. Tesis Ing. Forestal. Facultad de Ciencias Forestales, Universidad Austral de Chile. 2000. 73 p.

(31) MARAGAÑO, E. Efecto de las plantaciones de Pinus radiata $\mathrm{D}$. Don sobre el recurso agua en la localidad de Gualleco, zona de secano costero, VII Región. Tesis Ing. Forestal. Facultad de Ciencias Forestales, Universidad Austral de Chile, 2003. 55 p.

(32) BRECHTEL, H. Probleme beim Einsatz von Neutronensonde im Rahmen Hydrologisscher Messprogramme. Deutscher Verband fü Wasserwirtschaft und Kulturbau. Verlag Paul Parey. 1983. $301 \mathrm{p}$.

(33) OYARZUN, C., A. HUBER. Balance hídrico en plantaciones jóvenes de Eucalyptus globulus y Pinus radiata en el sur de Chile. Terra, 1999, vol. 17, No 1, p. 35-44.

(34) LONGPRE, M., Y. BERGERON, D. PARE. Effect of companion species on the growth of jack pine (Pinus banksiana Lamb.). Canadian Journal of Forest Research, 1994, No 24 , p. $1.846-1.853$.

(35) MOHAMMED, G. H., T. L. NOLAND, R. G. WAGNER Physiological perturbation in jack pine (Pinus banksiana Lamb.) in the presence of competing herbaceous vegetation. Forest Ecology and Management, 1998, $\mathrm{N}^{\circ} 103$, p. $77-85$ 